\title{
First near-relativistic solar electron events observed by EPD onboard Solar Orbiter
}

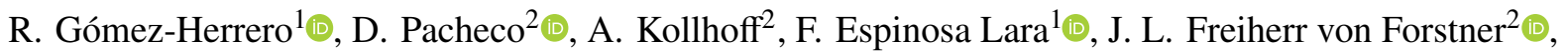

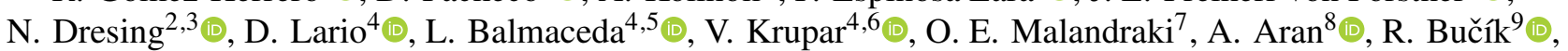 \\ A. Klassen ${ }^{2}$, K.-L. Klein ${ }^{10}$, I. Cernuda ${ }^{1}$, S. Eldrum ${ }^{2}$, H. Reid ${ }^{11} \odot$, J. G. Mitchell ${ }^{12,13}$, G. M. Mason ${ }^{14}$, G. C. Ho $^{14}$, \\ J. Rodríguez-Pacheco ${ }^{1}$, R. F. Wimmer-Schweingruber ${ }^{2}$, B. Heber ${ }^{2}$, L. Berger ${ }^{2}$, R. C. Allen ${ }^{14}$, N. P. Janitzek ${ }^{15}$, \\ M. Laurenza ${ }^{16}$, R. De Marco ${ }^{16}$, N. Wijsen ${ }^{17}$, Y. Y. Kartavykh ${ }^{18,19}$, W. Dröge ${ }^{19}$, T. S. Horbury ${ }^{20}$, M. Maksimovic ${ }^{10}$, \\ C. J. Owen ${ }^{11}$, A. Vecchio ${ }^{10,21}$, X. Bonnin ${ }^{10}$, O. Kruparova ${ }^{22}$, D. P1́ša ${ }^{22}$, J. Souček ${ }^{22}$, P. Louarn ${ }^{23}$, A. Fedorov ${ }^{23}$, \\ H. O’Brien ${ }^{20}$, V. Evans ${ }^{20}$, V. Angelini ${ }^{20}$, P. Zucca ${ }^{24}$, M. Prieto ${ }^{1}$, S. Sánchez-Prieto ${ }^{1}$, A. Carrasco ${ }^{1}$, J. J. Blanco ${ }^{1}$, \\ P. Parra ${ }^{1}$, O. Rodríguez-Polo ${ }^{1}$, C. Martín ${ }^{2,25}$, J. C. Terasa ${ }^{2}$, S. Boden ${ }^{2,26}$, S. R. Kulkarni ${ }^{2,27}$, A. Ravanbakhsh ${ }^{2,28}$, \\ M. Yedla ${ }^{2,28}$, Z. Xu ${ }^{2}$, G. B. Andrews ${ }^{14}$, C. E. Schlemm ${ }^{14}$, H. Seifert ${ }^{14}$, K. Tyagi ${ }^{14,29}$, W. J. Lees ${ }^{14}$, and J. Hayes ${ }^{14}$ \\ (Affiliations can be found after the references)
}

Received 10 November 2020 / Accepted 4 January 2021

\section{ABSTRACT}

Context. Solar Orbiter, launched in February 2020, started its cruise phase in June 2020, in coincidence with its first perihelion at 0.51 au from the Sun. The in situ instruments onboard, including the Energetic Particle Detector (EPD), operate continuously during the cruise phase enabling the observation of solar energetic particles.

Aims. In situ measurements of the first near-relativistic solar electron events observed in July 2020 by EPD are analyzed and the solar origins and the conditions for the interplanetary transport of these particles investigated.

Methods. Electron observations from keV energies to the near-relativistic range were combined with the detection of type III radio bursts and extreme ultraviolet (EUV) observations from multiple spacecraft in order to identify the solar origin of the electron events. Electron anisotropies and timing as well as the plasma and magnetic field environment were evaluated to characterize the interplanetary transport conditions.

Results. All electron events were clearly associated with type III radio bursts. EUV jets were also found in association with all of them except one. A diversity of time profiles and pitch-angle distributions was observed. Different source locations and different magnetic connectivity and transport conditions were likely involved. The July 11 event was also detected by Wind, separated 107 degrees in longitude from Solar Orbiter. For the July 22 event, the Suprathermal Electron and Proton sensor of EPD allowed for us to not only resolve multiple electron injections at low energies, but it also provided an exceptionally high pitch-angle resolution of a very anisotropic beam. This, together with radio observations of local Langmuir waves suggest a very good magnetic connection during the July 22 event. This scenario is challenged by a high-frequency occultation of the type III radio burst and a nominally non-direct connection to the source; therefore, magnetic connectivity requires further investigation.

Key words. acceleration of particles - Sun: particle emission - Sun: activity

\section{Introduction}

One of the science goals of the Solar Orbiter (SolO) mission is the analysis of the mechanisms by which solar eruptions produce energetic particle radiation that fills the heliosphere (Müller et al. 2020). This entails a deep understanding of how and where solar energetic particles (SEPs) are accelerated and how these particles are released and distributed into the interplanetary medium. SolO, which was launched on 2020 February 10 , enables in situ observations of SEPs from heliocentric distances $<1$ au, hence minimizing the transport effects undergone by the particles that often hinder the identification of their solar sources.

Solar electrons are closely associated with type III radio bursts and often with ${ }^{3} \mathrm{He}$-rich ion emissions (e.g., Wang et al. 2012). Due to their fast propagation, near-relativistic (NR) electrons are particularly useful for the identification of SEP sources at the Sun. There is a rich literature on the relationship between NR electron events and solar activity, with diverging results. While the early onset of electron events was reported to be closely related in time with the onset of radio emission and especially type III bursts produced by electron beams (Kallenrode \& Wibberenz 1991), later work from $1 \mathrm{au}$ frequently found significant delays in the electrons observed in space (Krucker et al. 1999; Haggerty \& Roelof 2002; Wang et al. 2016). There is also no simple relationship between energy spectra of electrons observed in space and thicktarget energy spectra derived from solar hard X-ray emission (Dröge et al. 1996; Krucker et al. 2007). The frequent detection of electrons at widely separated spacecraft $(\mathrm{s} / \mathrm{c})$ is a third issue that is not adequately understood, and it involves various transport processes in the corona and/or the heliosphere (Kallenrode et al. 1992; Wibberenz \& Cane 2006; Dresing et al. 2014; Gómez-Herrero et al. 2015; Xu et al. 2020; Zhang et al. 2021).

While HELIOS observations from the inner heliosphere addressed some of these questions (see Pacheco et al. 2019, and references therein), SolO provides a new view due to the better 


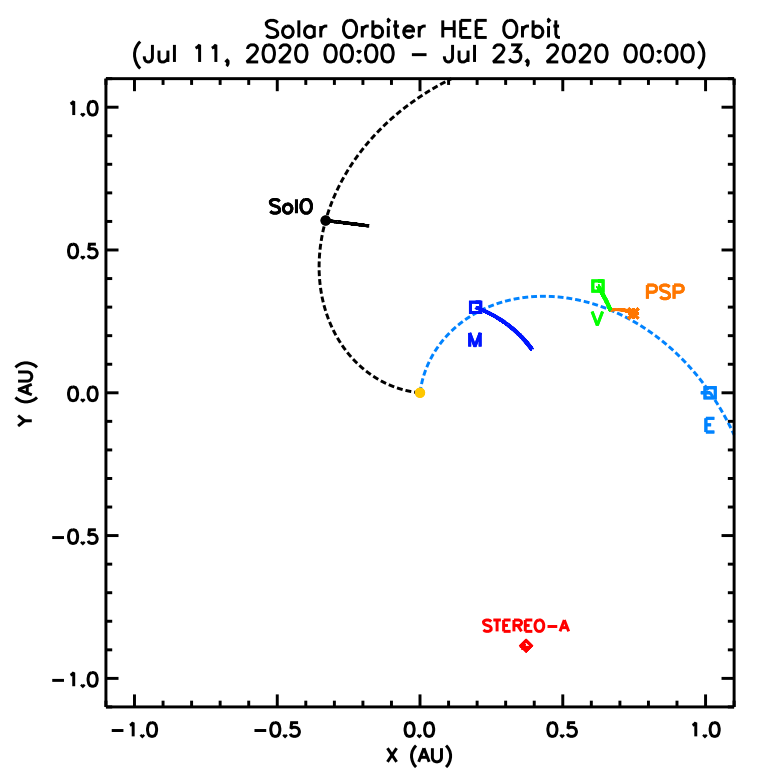

Fig. 1. Trajectories of SolO, the inner planets, STEREO-A, and Parker Solar Probe (PSP) during the period from 2020 July 11-23 (ecliptic plane projection, HEE frame). Nominal Parker spirals corresponding to a $300 \mathrm{~km} \mathrm{~s}^{-1}$ solar wind speed are shown for July 23.

coverage of the energy spectrum of the electrons and the existing complementary electromagnetic observations. The present Letter illustrates these potentialities with the first NR solar electron events observed by the Energetic Particle Detector suite (EPD, Rodríguez-Pacheco et al. 2020) from 2020 July 11-23, when SolO was at heliocentric distances between 0.61 and $0.69 \mathrm{au}$. Figure 1 shows the trajectory of SolO (black trace) in heliographic Earth ecliptic (HEE) coordinates. As shown by the idealized Parker spiral, SolO was magnetically connected to the solar backside (from Earth's point of view). We combined EPD electron observations, from $\mathrm{keV}$ energies to the NR range, with type III radio bursts observations by the Radio and Plasma Waves instrument (RPW, Maksimovic et al. 2020) and extreme ultraviolet (EUV) observations from STEREO-A and the near-Earth Solar Dynamics Observatory (SDO) to identify the solar origin of the in situ NR electron events.

\section{Observations and data analysis}

\subsection{NR electron events during the SolO cruise phase}

Although SolO started its cruise phase during the solar minimum between solar cycles 24 and 25, a number of NR electron events were observed in July 2020. Out of the four sensors of the EPD suite, this work uses measurements from the SupraThermal Electrons and Protons sensor (STEP) and the Electron Proton Telescope (EPT). STEP measures $4-80 \mathrm{keV}$ electrons and ions in a $28^{\circ} \times 54^{\circ}$ rectangular field of view (FOV) divided into 15 pixels. We note that STEP only provides reliable electron observations when the electron flux is significantly larger than the ion flux. EPT measures $20-400 \mathrm{keV}$ electrons and $20 \mathrm{keV}-15 \mathrm{MeV}$ ions using four telescopes in four different viewing directions, each with a $30^{\circ}$ conical FOV.

The first two panels of Fig. 2 show electron and ion intensity time profiles observed by the sunward-looking telescope of EPT and the fifteen-pixel-averaged electron and ion intensity time profiles measured by STEP at the lower energy range, from July $8-25$. The bottom panel shows the heliocentric distance and

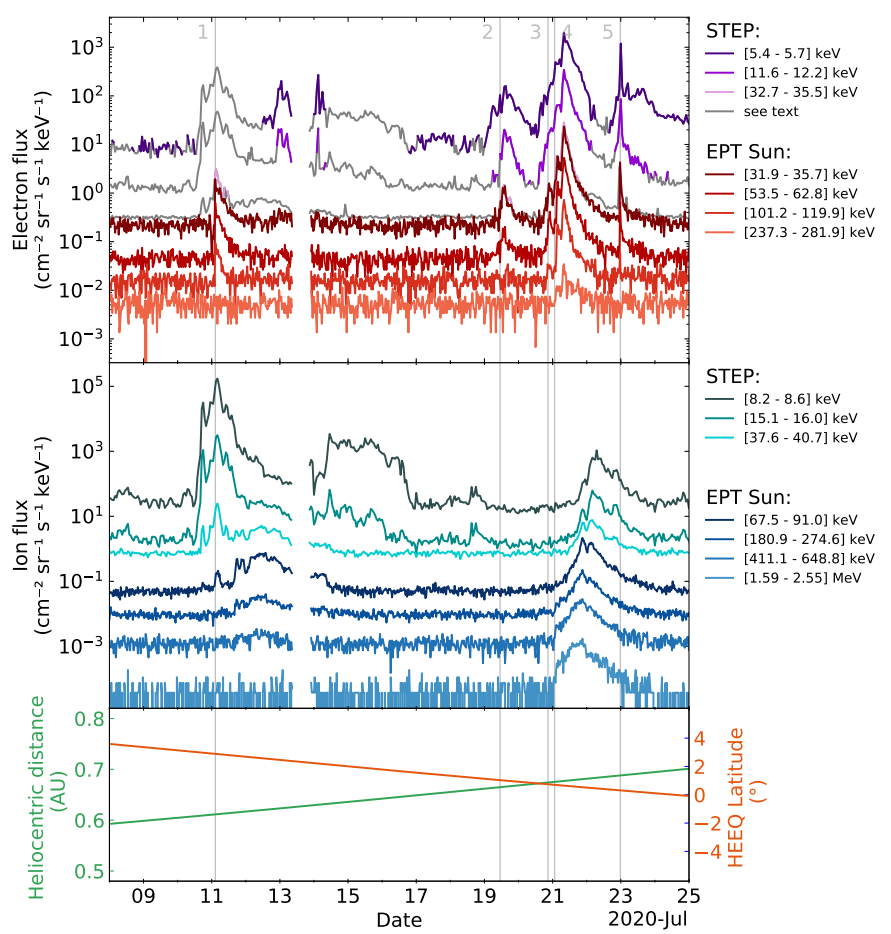

Fig. 2. Summary of electron and ion observations by the EPD/EPT and EPD/STEP instruments during July 2020. From top to bottom: (1) electron time profiles at seven different energy channels; (2) ion time profiles at seven different energy channels; and (3) heliographic latitude and radial distance. Gray lines indicate time periods without clear electron measurements and should be interpreted as an upper limit for the electron flux. The STEP electron fluxes can have large uncertainties if an increased ion flux was observed at the same time. Vertical lines mark the onset times of the five NR-electron enhancements listed in Table 1.

heliographic latitude of SolO. EPD observed five NR electron increases, some of them comprising several injections:

2020 July 11. The $58 \mathrm{keV}$ electron flux observed by EPT started to rise at 02:33 UT $\pm 5 \mathrm{~min}$. The electrons showed velocity dispersion in the energy range covered by EPT, but there was no significant increase above $\sim 170 \mathrm{keV}$. A low energy counterpart below $30 \mathrm{keV}$ could not be confirmed for this event because STEP measurements were dominated by ions (see second panel in Fig. 2). The event was observed by all the EPT telescopes; however, a moderate anisotropy was present during the rising phase, with higher fluxes observed by the EPT-Sun aperture, pointing sunward along the nominal Parker spiral direction (Fig. A.1). The electron increase was also observed near Earth by the 3DP instrument onboard Wind (Lin et al. 1995), located 107 degrees eastward from SolO. In spite of the significant difference in connectivity, Wind/3DP also observed moderate electron anisotropies (Fig. A.4).

2020 July 19. The $58 \mathrm{keV}$ electron flux showed a gradual rise starting at 11:00 UT $\pm 1 \mathrm{~h}$. This event measured by STEP and EPT was not visible above $70 \mathrm{keV}$. No significant anisotropies nor velocity dispersion were observed, but the observational statistics were limited by the small size of the event.

2020 July 20. The $58 \mathrm{keV}$ electron flux started to rise at 20:40 UT $\pm 10 \mathrm{~min}$. The highest intensity and earliest onset were observed by the northward-looking EPT telescope, pointing closer to pitch-angle $0^{\circ}$ (Fig. A.2). No significant increase was observed above $\sim 100 \mathrm{keV}$. 
Table 1. Electron events observed by EPD during July 2020.

\begin{tabular}{|c|c|c|c|c|c|}
\hline Event & 1 & 2 & 3 & 4 & 5 \\
\hline Date & July 11 & July 19 & July 20 & July 21 & July 22 \\
\hline Onset (UT) ${ }^{(a)}$ & $02: 33 \pm 5 \mathrm{~min}$ & $11: 00 \pm 1 \mathrm{~h}$ & $20: 40 \pm 10 \mathrm{~min}$ & $01: 25 \pm 10 \mathrm{~min}$ & $23: 40 \mathrm{UT} \pm 10 \mathrm{~min}$ \\
\hline Type III (UT) ${ }^{(b)}$ & $02: 21$ & $10: 27$ & $20: 13$ & $01: 01^{(c)}$ & $23: 32$ \\
\hline EUV jet (UT) & $02: 21$ & no & $20: 20-20: 56^{(d)}$ & $01: 16-01: 36^{(d)}$ & $23: 33$ \\
\hline EUV jet observer & SDO/AIA & N/A & STA/EUVI & STA/EUVI & STA/EUVI \\
\hline EUV jet PA $\left(^{\circ}\right)^{(e)}$ & $\sim 302$ & $\mathrm{~N} / \mathrm{A}$ & $\sim 70$ & $\sim 70$ & $\sim 70$ \\
\hline$V_{\mathrm{sw}}(\mathrm{km} / \mathrm{s})$ & No data & 264 & 284 & 275 & 320 \\
\hline$\phi_{\text {Parker }}(\mathrm{CL})$ & $204^{(f)}$ & 121 & 100 & 100 & 69 \\
\hline Remarks & & & & Multiple injections & Multiple injections \\
\hline
\end{tabular}

Notes. ${ }^{(a)}$ Determined at $58 \mathrm{keV}$ using the telescope showing earliest onset. For multiple injections, the first onset is listed. ${ }^{(b)}$ Onset at $2 \mathrm{MHz}$ on SolO/RPW data for events $1,3,4$, and 5 and Wind/Waves for event 2. ${ }^{(c)}$ Series of type III bursts along the day. ${ }^{(d)}$ Series of jets. ${ }^{(e)}$ Position angle of the jet(s) observed over the solar limb, counter-clockwise from the solar north pole. ${ }^{(f)}$ No SWA data are available, footpoint was obtained assuming $V_{\mathrm{sw}}=350 \mathrm{~km} \mathrm{~s}^{-1}$.

2020 July 21. This event comprises at least three consecutive, partly overlapping electron injections, with electron onsets at 01:25, 03:05, and 06:25 UT $\pm 10 \mathrm{~min}$ (determined at $58 \mathrm{keV}$ ). The increases were progressively more intense and energetic, reaching $\sim 1 \mathrm{MeV}$ (observed by the High Energy Telescope (HET) of EPD, not shown). The peak flux was reached at $\sim 08: 30$ UT on July 21 and the decay phase lasted until mid-July 22. EPT directional intensities revealed measurable anisotropies, but smaller than during the previous event (Fig. A.2).

2020 July 22. EPT observed a very anisotropic electron event reaching energies slightly above $100 \mathrm{keV}$. The spike-like time profile, similar to the events described by Klassen et al. (2011), showed a sharp onset with clear velocity dispersion, starting at 23:40 UT $\pm 10 \mathrm{~min}$ at $58 \mathrm{keV}$. This period was cleanly observed by STEP at lower energies, which revealed additional spike-like injections preceding and following the event observed by EPT. The EPD pitch-angle coverage for this event was nearly optimal (Figs. A.3 and A.5.).

The events on July 19, 20, 21, and probably July 22 resulted in an extended period with observable ${ }^{3} \mathrm{He}$ fluxes and enhanced ${ }^{3} \mathrm{He} /{ }^{4} \mathrm{He}$ ratios measured by the Suprathermal Ion Spectrograph (EPD/SIS), which spanned from mid-July 19 to July 25 (cf. Fig. 3 in Mason et al. 2021). Anisotropies during the events are discussed in further detail in Appendix A. In situ magnetic field and plasma observations by the Magnetometer (MAG, Horbury et al. 2020) and the Solar Wind Analyser suite (SWA, Owen et al. 2020) onboard SolO are presented and discussed in Appendix B.

The main observational characteristics of the NR electron events are summarized in Table 1 . The first row numbers the events in sequential order. The event date and the observed onset time at $58 \mathrm{keV}$ are listed in the second and third row, respectively. The timing of type III radio bursts and coronal EUV jets likely associated with each event are listed in the fourth and fifth row, respectively (see Sects. 2.2 and 2.3 for further details). The sixth and seventh row describe the s/c observing the EUV jet and its approximate position angle (PA, counter-clockwise from the solar north), respectively. The times listed for the type III radio bursts and the EUV jets are the times at which these phenomena were observed locally by the listed s/c. The eighth row shows the 1-hour averaged solar wind speed at the onset of the NR electron event (see Appendix B). This value was used to estimate the Carrington Longitude (CL) of the footpoint of the s/c along an ideal

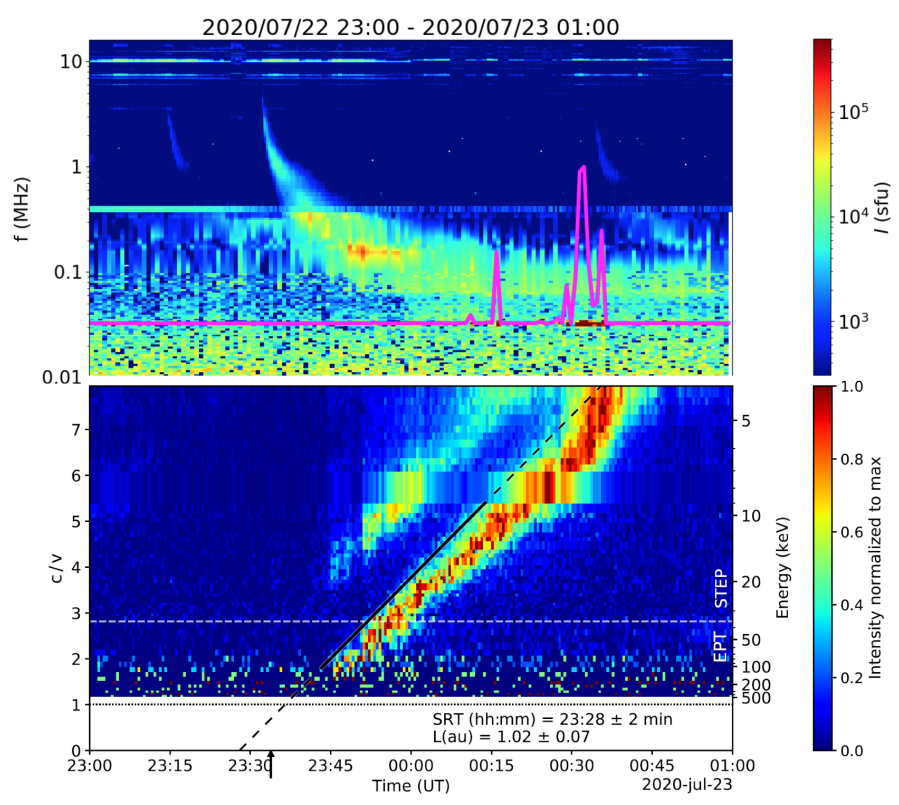

Fig. 3. Top: SolO/RPW radio dynamic spectrum observed in association with the electron event with an onset in late July 22 . The magenta line is the spectral flux at the local plasma frequency, where the peaks indicate locally generated Langmuir waves. Bottom: electron $c / v$ versus time plot. The color scale corresponds to the particle intensity, normalized to the maximum value for each energy (speed) channel. The black line is a linear fit to electron arrival times, indicating the solar particle release time. The energy interval used for the fit is marked with a solid line (see text for details).

Parker spiral (ninth row). Some additional remarks are described in the last row.

The bottom panel in Fig. 3 shows an inverse-speed versus time plot (typically used for velocity dispersion analysis) for the July 22 event, combining STEP and EPT electron data. The color scale represents the intensity, normalized to the maximum value for each energy channel. The dashed white line separates STEP and EPT observations. Two different injections were resolved at low energies, both of them showing clear velocity dispersion and very narrow time profiles over the whole energy range.

The black slanted line corresponds to a linear fit of the onset times at each energy bin above $9 \mathrm{keV}$ for the second injection. The onset times are defined as the first time when the flux, at $30 \mathrm{~s}$ resolution, is higher than the pre-event average plus 2.5 
standard deviations and stays above that threshold for at least two consecutive points. The linear fit provides an estimation of the effective path length $L$ and the solar release time (SRT) at the Sun. Two-sigma errors on the fit parameters were estimated repeating the fit with random samples of half of the data points. The lowest energy electrons excluded from the fit are probably influenced by instrumental effects (Gómez-Herrero et al. 2017, and references therein) or have modified distributions due to wave-particle interactions with the Langmuir waves detected co-temporally (e.g., Reid \& Kontar 2018) and they would arrive at Solar Orbiter early (Kontar \& Reid 2009).

The theoretical Parker spiral length connecting to SolO for a solar wind speed of $320 \mathrm{~km} \mathrm{~s}^{-1}$ is $0.77 \mathrm{au}$, which is substantially shorter than the value obtained from the fit ( $L=1.02 \mathrm{au}$ ). The black arrow at 23:34 UT marks the SRT shifted by the light propagation time to SolO in order to allow a direct comparison with electromagnetic observations from $0.69 \mathrm{au}$. The top panel in Fig. 3 shows the radio dynamic spectrum observed by SolO/RWP during the same time interval. A type III radio burst was observed by RPW, with a start time at 23:32 UT (value determined at $2 \mathrm{MHz}$ ), meaning that the inferred electron release time and the type III radio burst differ only by 2 min (see Sect. 2.2).

\subsection{Radio observations}

Radio dynamic spectra from STEREO/SWAVES (Bougeret et al. 2008), Wind/Waves (Bougeret et al. 1995), and SolO/RPW were examined for the period under analysis. Type III radio bursts were found in association with all the electron events presented here (Table 1). None of the electron events were accompanied by type II radio bursts.

The top panel of Fig. 3 shows the dynamic spectrum observed by SolO/RPW during the electron event starting in late July 22. The magenta line shows the spectral flux around the local plasma frequency. The distinct peaks are due to Langmuir waves locally generated when the electron beam causing the type III radio emission reached the s/c location. We note the coincidence of the intensity increase with the detection of few $\mathrm{keV}$ electrons by STEP. These observations support the existence of a direct magnetic connection to the solar source of the electrons (e.g., Lin 1990) in this event, also observed in the events on July 20 and 21 (see details in Krupař et al., in prep.).

The attenuation of the type III burst observed at high frequencies suggests the source was partially occulted as viewed from SolO, while STEREO-A saw it entirely (not shown). Furthermore, the lack of a clear type III detection from PSP (not shown) places the most probable location near the east limb from STEREO-A and behind the west limb from SolO (for reference, see Fig. 1).

The type III burst seen by STEREO-A/SWAVES corresponds to fundamental emission, while SolO/RPW observed harmonic emission. According to Thejappa et al. (2012), fundamental emission in type III radio bursts is more directive than the second harmonic, which explains why SolO observed the weaker second harmonic emission, while STEREO-A, with direct visibility of the source, observed the fundamental emission.

\subsection{Associated solar activity and source locations}

During the period under analysis, remote-sensing instruments onboard SolO were not active. EUV and coronagraph observations from SDO/AIA (Lemen et al. 2012), SOHO/ LASCO (Brueckner et al. 1995), and STEREO-A/SECCHI
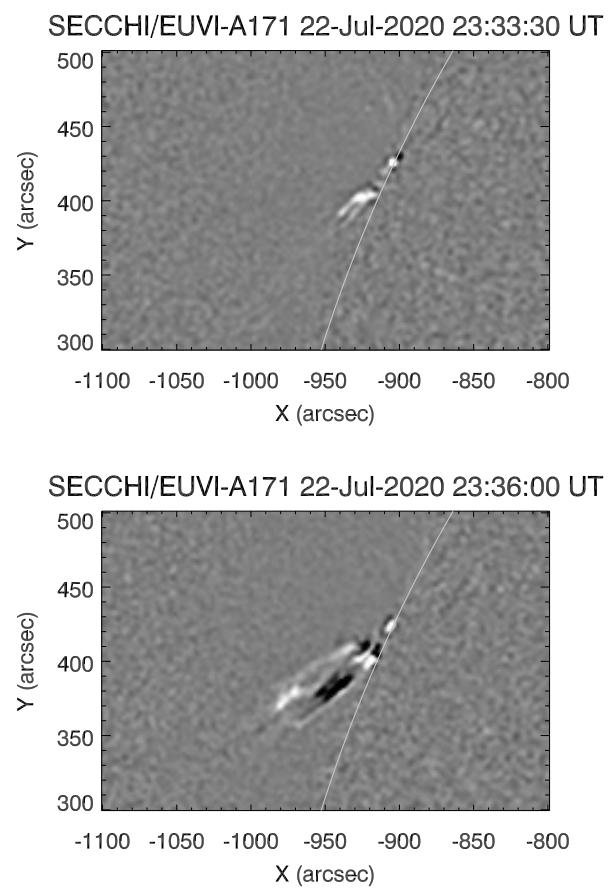

Fig. 4. STEREO/SECCHI EUVI $171 \AA$ r running difference images showing a jet over the NE limb in coincidence with the type III radio burst observed on July 22 23:33 UT.

(Howard et al. 2008) have been examined in order to explore solar activity phenomena and source regions potentially associated with the electron events observed by EPD.

For all the events except the weak event on July 19, sequences of multiple narrow ejections were found in 171, 195, and $304 \AA$ EUV images, with appearance times very close to the in situ electron onsets. None of the events were associated with large CMEs. Some small flux-rope ejections were seen, but there was no time coincidence with the electron events analyzed here.

The times, locations, and observing s/c of the jets are listed in Table 1. They were observed above the west limb on SDO (July 11) and the east limb from STEREO-A (July 20, 21, and 22). When comparing the observation times of these EUV jets at $1 \mathrm{au}$ and the type III radio bursts at SolO, it should be taken into account that the light travel time to STEREO-A and SDO is 2-3 min longer than to SolO.

Figure 4 shows two STEREO-A/EUVI running difference images at $171 \AA$, around the time of the type III radio burst associated with the July 22 event. We note the agreement with the timing of the type III radio burst and the electron release time inferred from a velocity dispersion analysis (Fig. 3). The jet showed a strongly non-radial propagation trajectory toward the equator (projected onto the plane of the sky), likely reflecting the non-radial magnetic field configuration around the source region, as described by Klassen et al. (2018) and Bučík et al. (2018).

\section{Summary and conclusions}

In spite of the solar minimum conditions, after its first perihelion, SolO detected several NR electron events during 2020 July 11-24. All of them were clearly associated with type III radio bursts observed by multiple s/c. EUV jets were observed above the east limb from STEREO-A for the July 20, 21, and 22 events and above the west limb from SDO for the July 11 event, 
with good time coincidence with the type III radio bursts. While velocity dispersion analysis results should always be treated with caution (e.g., Lintunen \& Vainio 2004; Sáiz et al. 2005; Kahler \& Ragot 2006), the results presented here suggest that this method provides a reasonable estimation of the solar release time for SEP events in the inner heliosphere under low-scattering conditions. As commonly happens for impulsive SEP events, $\mathrm{EPD} / \mathrm{SIS}$ detected several ${ }^{3} \mathrm{He}$ and heavy ion enhancements during the same time interval (Mason et al. 2021). The period was not disturbed by major CMEs nor shocks (Appendix B).

Examination of NR electron observations from instruments onboard STEREO, near-Earth s/c, and PSP for all the events revealed that only the July 11 event (Fig. A.1) was clearly observed by Wind/3DP (Fig. A.4), separated 107 degrees in longitude from SolO. Electron fluxes observed at Wind and SolO showed significant anisotropies, providing an interesting candidate for further investigation of the interplanetary transport conditions causing the wide electron spread during some impulsive events (e.g., the possible role of the field line spread in the corona, interplanetary field-line meandering, and cross-field diffusion). On the other hand, while SolO detected ${ }^{3} \mathrm{He}$ for this event, this was not the case near Earth (Mason et al. 2021). This may reflect that ${ }^{3} \mathrm{He}$ was below the detection limit at Earth or be an indication of a narrowed spread of energetic ions compared to electrons.

At the end of July 22, EPT detected a very anisotropic and short duration event with clear velocity dispersion (Figs. 3 and A.3). At low energies, additional injections showing similar characteristics were revealed by STEP. The spike-like time profiles, prompt onsets, and beamed pitch-angle distributions (PADs), together with the in situ detection of Langmuir waves by RPW support a very good magnetic connection to the source. However, although STEREO-A observed a jet at the east solar limb, the nominal magnetic footpoint of SolO along the ideal Parker spiral was located more than 30 degrees behind the east limb from the STEREO-A point of view. There was good time coincidence of the type III radio burst, the jet, and the inferred electron release time. The southward trajectory of the jet suggests non-radial coronal magnetic field lines which may help to explain how the narrow beam of energetic electrons reached IMF lines connecting to SolO at 0.69 au. However, further investigation is required to clarify the magnetic connectivity and interplanetary propagation for this electron event.

The series of impulsive electron events presented here shows a rich variety of behavior regarding time profiles and PADs, which range from almost isotropic to beam-like, suggesting changing interplanetary transport conditions and/or magnetic connectivity in the course of a few days. The high quality of the near-relativistic electron data provided by EPT, the good directional resolution of STEP down to few $\mathrm{keV}$ energies, the broad spectral range obtained combining both sensors, with excellent energy resolution, and the high time cadence ensure that future observations close to the Sun will contribute to the understanding of the acceleration, release, and transport processes of energetic particles. EPD observations will play a key role in the identification of the sources of impulsive events and the links between the NR electrons and the ion populations enriched in ${ }^{3} \mathrm{He}$ and heavy ions, which often, but not always, concur during impulsive SEP events.

Acknowledgements. The UAH team acknowledges the financial support by the Spanish MICIU under projects ESP2017-88436-R and PID2019-104863RBI00/AEI/10.13039/501100011033. The CAU Kiel team acknowledges support by the German Federal Ministry for Economic Affairs and Energy, the
German Space Agency (Deutsches Zentrum für Luft- und Raumfahrt e.V., DLR) under grants 50OT0901, 50OT1202, 50OT1702, 50OT2002, and 50OC1702. A. Aran acknowledges the support by the Spanish Ministerio de Ciencia e Innovación (MICINN) under grant PID2019-105510GB-C31 and through the "Center of Excellence María de Maeztu 2020-2023" award to the ICCUB (CEX2019-000918-M). The RPW team thanks the French space agency CNES, the Center National de Recherches Scientifiques, the Paris Observatory, the Swedish National Space agency, ESA-PRODEX and the Czech Science Foundation (17-08772S) for the funding provided. V. Krupar acknowledges the support by NASA under grants 18-2HSWO218_2-0010 and 19-HSR-19_2-0143. The Solar Orbiter magnetometer was funded by the UK Space Agency (grant ST/T001062/1). Solar Orbiter Solar Wind Analyser (SWA) data are derived from scientific sensors which have been designed and created, and are operated under funding provided in numerous contracts from the UK Space Agency (UKSA, most recently grant ST/T001356/1), the UK Science and Technology Facilities Council (STFC), the Agenzia Spaziale Italiana (ASI), the Centre National d'Etudes Spatiales (CNES, France), the Centre National de la Recherche Scientifique (CNRS, France), the Czech contribution to the ESA PRODEX programme and NASA.

\section{References}

Bougeret, J.-L., Kaiser, M. L., Kellogg, P. J., et al. 1995, Space Sci. Rev., 71, 231 Bougeret, J. L., Goetz, K., Kaiser, M. L., et al. 2008, Space Sci. Rev., 136, 487 Brueckner, G. E., Howard, R. A., Koomen, M. J., et al. 1995, Sol. Phys., 162, 357

Bučík, R., Innes, D. E., Mason, G. M., et al. 2018, ApJ, 852, 76

Diego, P., Piersanti, M., Laurenza, M., \& Villante, U. 2020, J. Geophys. Res. (Space Phys.), 125, e28281

Dresing, N., Gómez-Herrero, R., Heber, B., et al. 2014, A\&A, 567, A27

Dröge, W. 1996, in High Energy Solar Physics, eds. R. Ramaty, N. Mandzhavidze, AIP Conf. Ser., 374, 78

Gómez-Herrero, R., Dresing, N., Klassen, A., et al. 2015, ApJ, 799, 55

Gómez-Herrero, R., Dresing, N., Klassen, A., et al. 2017, ApJ, 840, 85

Gosling, J. T., Hildner, E., Asbridge, J. R., Bame, S. J., \& Feldman, W. C. 1977,

J. Geophys. Res., 82, 5005

Haggerty, D. K., \& Roelof, E. C. 2002, ApJ, 579, 841

Horbury, T. S., O’Brien, H., Carrasco Blazquez, I., et al. 2020, A\&A, 642, A9

Howard, R. A., Moses, J. D., Vourlidas, A., et al. 2008, Space Sci. Rev., 136, 67 Kahler, S., \& Ragot, B. R. 2006, ApJ, 646, 634

Kallenrode, M. B., \& Wibberenz, G. 1991, ApJ, 376, 787

Kallenrode, M. B., Cliver, E. W., \& Wibberenz, G. 1992, ApJ, 391, 370

Klassen, A., Gómez-Herrero, R., \& Heber, B. 2011, Sol. Phys., 273, 413

Klassen, A., Dresing, N., Gómez-Herrero, R., Heber, B., \& Veronig, A. 2018, A\&A, 614, A61

Kontar, E. P., \& Reid, H. A. S. 2009, ApJ, 695, L140

Krucker, S., Larson, D. E., Lin, R. P., \& Thompson, B. J. 1999, ApJ, 519, 864

Krucker, S., Kontar, E. P., Christe, S., \& Lin, R. P. 2007, ApJ, 663, L109

Lemen, J. R., Title, A. M., Akin, D. J., et al. 2012, Sol. Phys., 275, 17

Lin, R. P. 1990, in Basic Plasma Processes on the Sun, eds. E. R. Priest, \& V. Krishan, IAU Symp., 142, 467

Lin, R. P., Anderson, K. A., Ashford, S., et al. 1995, Space Sci. Rev., 71, 125

Lintunen, J., \& Vainio, R. 2004, A\&A, 420, 343

Maksimovic, M., Bale, S. D., Chust, T., et al. 2020, A\&A, 642, A12

Mason, G. M., Ho, G. C., Allen, R. C., et al. 2021, A\&A, 656, L1 (SO Cruise Phase SI)

Müller, D., St. Cyr, O. C., Zouganelis, I., et al. 2020, A\&A, 642, A1

Owen, C. J., Bruno, R., Livi, S., et al. 2020, A\&A, 642, A16

Pacheco, D., Agueda, N., Aran, A., Heber, B., \& Lario, D. 2019, A\&A, 624, A3

Reid, H. A. S., \& Kontar, E. P. 2018, ApJ, 867, 158

Rodríguez-Pacheco, J., Wimmer-Schweingruber, R. F., Mason, G. M., et al. 2020, A\&A, 642, A7

Sáiz, A., Evenson, P., Ruffolo, D., \& Bieber, J. W. 2005, ApJ, 626, 1131

Thejappa, G., MacDowall, R. J., \& Bergamo, M. 2012, ApJ, 745, 187

Wang, L., Lin, R. P., Krucker, S., \& Mason, G. M. 2012, ApJ, 759, 69

Wang, L., Krucker, S., Mason, G. M., Lin, R. P., \& Li, G. 2016, A\&A, 585, A119

Wibberenz, G., \& Cane, H. V. 2006, ApJ, 650, 1199

Xu, Z., Guo, J., Wimmer-Schweingruber, R. F., et al. 2020, ApJ, 902, L30

Zhang, J., Temmer, M., Gopalswamy, N., et al. 2021, Prog. Earth Planet. Sci., 8, 56

1 Universidad de Alcalá, Space Research Group, 28805 Alcalá de Henares, Spain e-mail: raul.gomezh@uah.es

2 Institut für Experimentelle und Angewandte Physik, ChristianAlbrechts-Universität zu Kiel, Kiel, Germany 
3 Now at Department of Physics and Astronomy, University of Turku, 20014 Turku, Finland

${ }^{4}$ Heliophysics Science Division, NASA Goddard Space Flight Center, Greenbelt, MD, USA

5 George Mason University, Fairfax, VA, USA

${ }^{6}$ Goddard Planetary Heliophysics Institute, University of Maryland, Baltimore County, Baltimore, MD 21250, USA

7 National Observatory of Athens, Institute for Astronomy, Astrophysics, Space Applications and Remote Sensing, Athens, Greece

8 Departament de Física Quàntica i Astrofísica, Institut de Ciències del Cosmos (ICCUB), Universitat de Barcelona (UB-IEEC), Barcelona, Spain

9 Southwest Research Institute, 6220 Culebra Rd, San Antonio, TX 78238, USA

10 LESIA, Observatoire de Paris, Université PSL, CNRS, Sorbonne Université, Université de Paris, 5 place Jules Janssen, 92195 Meudon, France

11 Department of Space and Climate Physics, University College London, Holmbury St Mary, Dorking, RH5 6NT, UK

12 Department of Physics, George Washington University, Washington, DC 20052, USA

13 NASA Goddard Space Flight Center, Greenbelt, MD 20771, USA

14 Johns Hopkins University Applied Physics Laboratory, Laurel, MD, USA

15 ESA-ESAC, Madrid, Spain European Space Agency (ESA/ESAC), Villafranca del Castillo, 28692 Madrid, Spain
${ }^{16}$ INAF Istituto di Astrofisica e Planetologia Spaziale, Via Fosso del Cavaliere 100, 00133 Roma, Italy

17 Department of Mathematics/Centre for mathematical Plasma Astrophysics, KU Leuven, Belgium

18 Ioffe Physical-Technical Institute, St. Petersburg 194021, Russia

19 Institute for Theoretical Physics and Astrophysics, University of Würzburg, Würzburg, Germany

20 Department of Physics, Imperial College London, London SW7 2AZ, UK

${ }^{21}$ Radboud Radio Lab, Department of Astrophysics/IMAPP-Radboud University, PO Box 9010, 6500 GL Nijmegen, The Netherlands

22 Institute of Atmospheric Physics of the Czech Academy of Sciences, Prague, Czech Republic

23 Institut de Recherche en Astrophysique et Planétologie, 9, Avenue du Colonel ROCHE, BP 4346, 31028 Toulouse Cedex 4, France

24 ASTRON-The Netherlands Institute for Radio Astronomy, Oude Hoogeveensedijk 4, 7991 PD Dwingeloo, The Netherlands

25 Now at German Aerospace Center (DLR), Department of Extrasolar Planets and Atmospheres, Berlin, Germany

26 Now at DSI Datensicherheit GmbH, Rodendamm 34, 28816 Stuhr, Germany

27 Now at Deutsches Elektronen-Synchrotron (DESY), Platanenallee 6, 15738 Zeuthen, Germany

28 Now at Max-Planck-Institute for Solar System Research, Justusvon-Liebig-Weg 3, 37077 Göttingen, Germany

29 Now at University of Colorado/LASP, Boulder, CO, USA 


\section{Appendix A: Electron anisotropies}

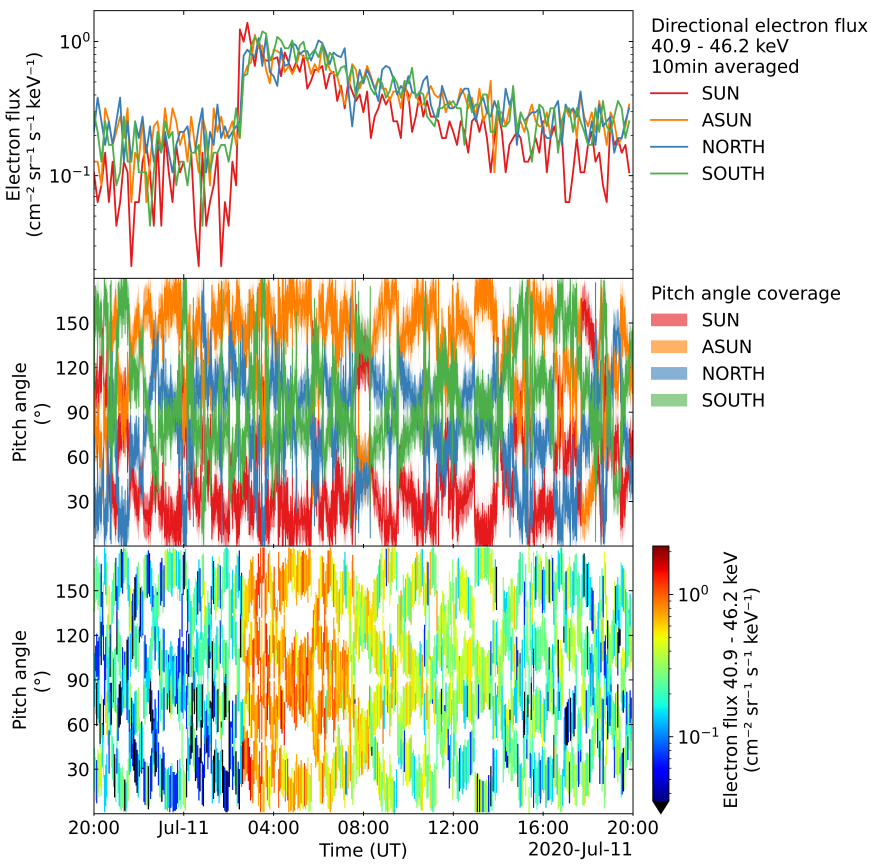

Fig. A.1. Time evolution of directional electron intensity measurements during the July 11 event. From top to bottom: (1) $40.9-46.2 \mathrm{keV}$ electron intensities observed by the four EPD apertures; (2) pitch-angle coverage corresponding to each aperture; and (3) pitch angle distribution with color-coded intensity for the same energy range shown in the first panel.

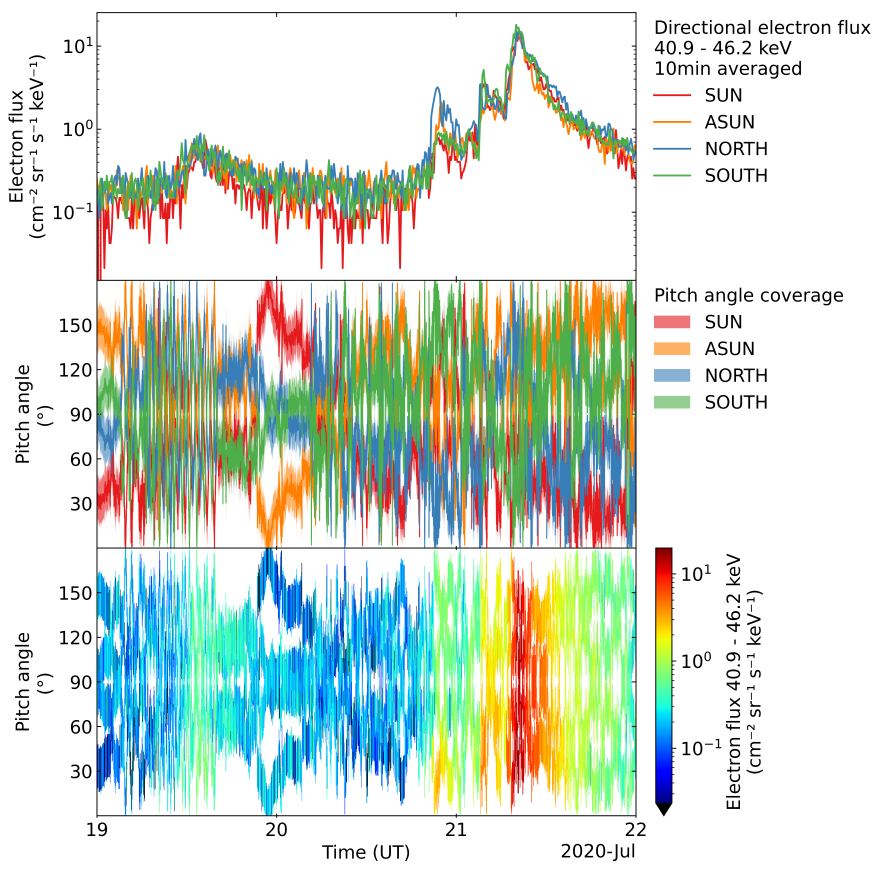

Fig. A.2. Time evolution of directional electron intensity measurements during the July 19-21 event series. The panels are the same as in Fig. A.1.

Directional intensities from EPT telescopes can be combined with the magnetic field vector data in order to produce electron PADs. The PAD quality for a particular event (i.e., the coverage of the pitch-angle space) is conditioned by the relative

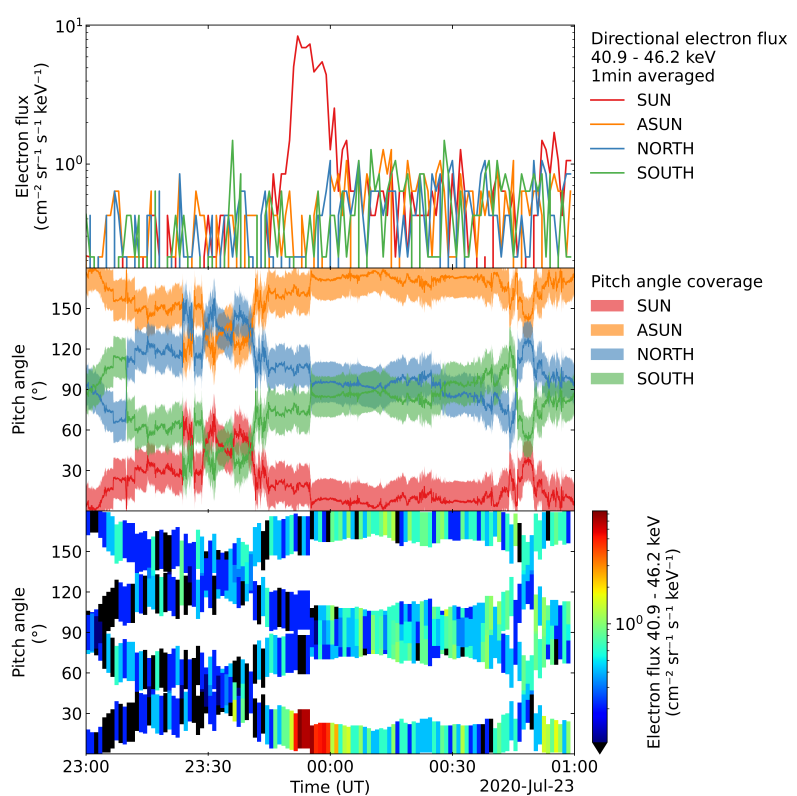

Fig. A.3. Time evolution of directional electron intensity measurements during the July 22 event. The panels are the same as in Fig. A.1.

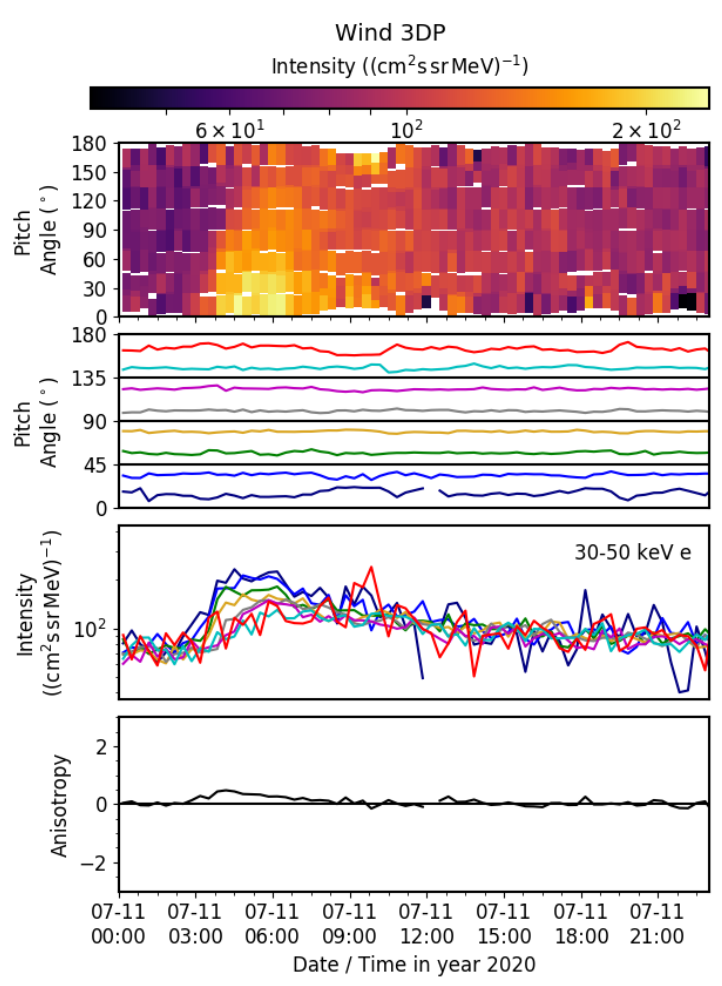

Fig. A.4. Summary of Wind/3DP observations of 30-50 keV electrons during the electron event on July 11. From top to bottom: electron PAD, pointing for each pitch-angle bin, intensities observed by each pitch-angle bin, and anisotropy index (calculated as described in Dresing et al. 2014).

orientation of the magnetic field vector and the sensor apertures. Figures A.1-A.3 summarize the $40.9-46.2 \mathrm{keV}$ electron anisotropy information provided by EPT for the July 11, July 19-21, and July 22 events, respectively. We note that on July 19, EPT pitch-angle coverage goes through sharp and periodic changes, coinciding with most of event 2 due to s/c rotations. 


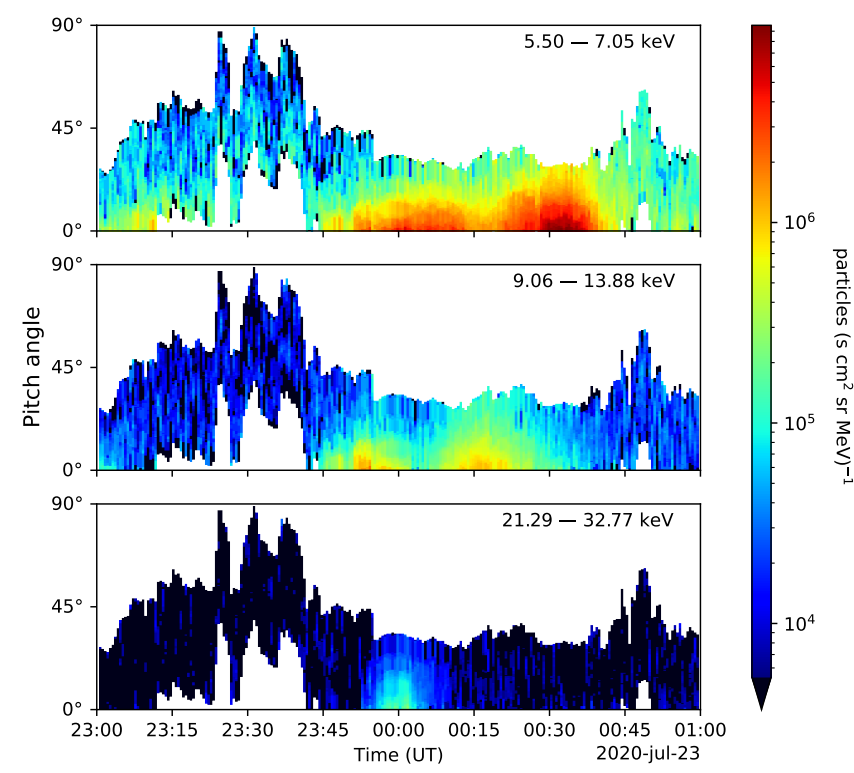

Fig. A.5. Electron PAD observed by STEP at three different energy bands during the July 22 event. The time scale is the same as the one used in Figs. 3 and A.1. We note that in this case, the $Y$-axis covers the interval $0-90^{\circ}$.

Figure A.4 shows the evolution of the PAD, directional intensities, and anisotropy index of 30-50 keV electrons observed by Wind/3DP during the July 11 event.

Moreover, the pixelated FOV of STEP can provide improved angular resolution for a limited range of pitch angles. Fig. A.5 shows the electron pitch-angle distribution obtained from STEP data for three different energy ranges during the July 22 event. For this event, EPT and STEP observed very anisotropic fluxes, which were resolved in two different beams at low energies (Fig. 3). The interplanetary magnetic field and solar wind observations showed very stable conditions during the whole interval and during most of the event the magnetic field vector was almost aligned with the STEP and the EPT-Sun sensor apertures, providing a nearly optimal pitch-angle coverage of the two narrow field-aligned electron beams observed by STEP below $30 \mathrm{keV}$. We note that before 23:40 UT, the electron beam was not aligned with the FOVs, which is probably the cause of the abrupt start of the first electron beam, and hinders the detection of the higher energy electrons by EPT, in case they were present.

\section{Appendix B: Interplanetary context}

For the same period shown in Fig. 2 and B.1 shows from top to bottom the solar wind proton speed $\left(V_{\mathrm{p}}\right)$, density $\left(n_{\mathrm{p}}\right)$, and temperature $\left(T_{\mathrm{p}}\right)$ as measured by SWA and the interplanetary magnetic field (IMF) magnitude $\left(B_{\mathrm{IMF}}\right)$ and direction in the s/c centered radial tangential normal (RTN) coordinates (polar angle $\theta_{\mathrm{IMF}}$ and azimuthal angle $\phi_{\mathrm{IMF}}$ ) as measured by MAG. The vertical lines indicate the onset time of the NR electron events listed in Table 1. SWA observations were only available for the last part of the period, showing predominantly slow solar wind conditions.

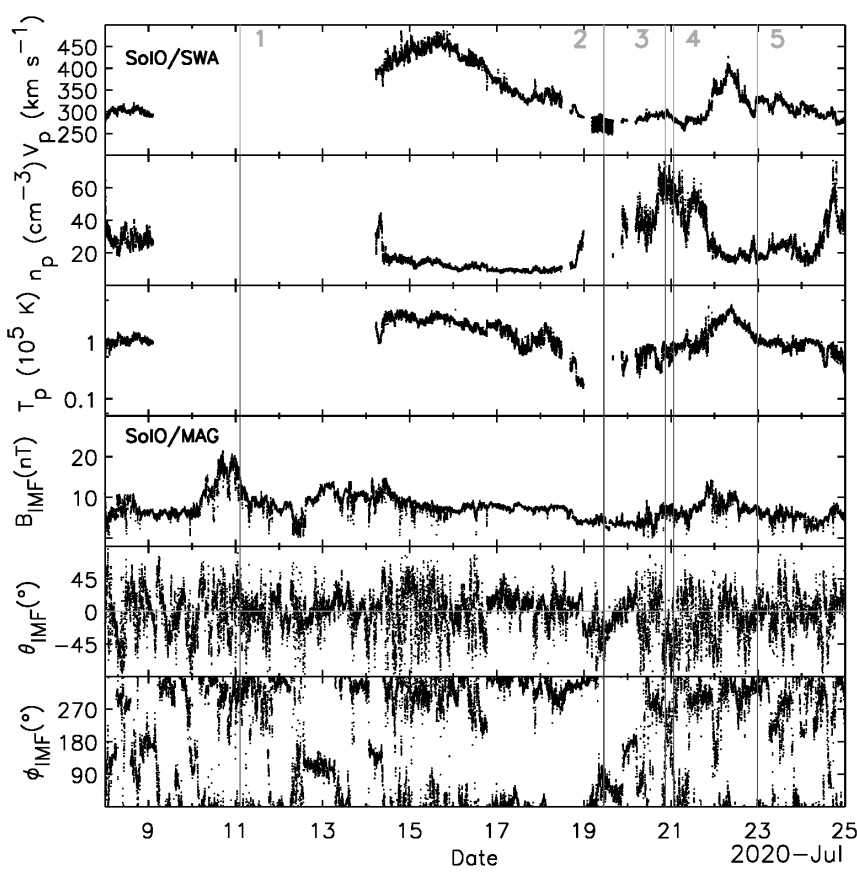

Fig. B.1. Summary of the solar wind and interplanetary magnetic field measurements by the SWA/PAS and MAG instruments in July 2020. From top to bottom: solar wind speed, density, and temperature as well as IMF intensity, and IMF polar and azimuthal angles in RTN coordinates. Vertical lines mark the onset times of the five NR-electron enhancements listed in Table 1.

The onset of event 1 occurred after the passage of a compressed magnetic field without clear shock discontinuities at its boundaries. The occurrence of a low-energy ion intensity enhancement in coincidence with this IMF structure as observed by both STEP and EPT (Fig. 2), with the suprathermal proton enhancement seen earlier than the more energetic ions detected by EPT, suggests that this IMF enhancement is most likely the signature of a stream interaction region (SIR).

The onset of event 2 occurred during s/c rolling maneuvers (from 4:00 UT to 16:00 UT on July 19) that affected SWA data, exhibiting large oscillations in density and temperature, which were removed from Fig. B.1. Nevertheless, event 2 developed during a period characterized by low $T_{\mathrm{p}}$ and low $V_{\mathrm{p}}\left(<270 \mathrm{~km} \mathrm{~s}^{-1}\right)$ embedded between the decay of the fast $\left(\sim 450 \mathrm{~km} \mathrm{~s}^{-1}\right)$ stream (observed earlier on July 15) and an approaching solar wind stream characterized by enhanced values of $n_{\mathrm{p}}$ and low values of $V_{\mathrm{p}}, T_{\mathrm{p}}$, and $B_{\mathrm{IMF}}$. The onset of events 3 and 4 occurred during this period and in coincidence with IMF rotations. These conditions suggest that a non-compressive density enhancement reached SolO, indicating a possible small eruption from the Sun (Gosling et al. 1977; Diego et al. 2020). The passage of a SIR-like magnetic structure started on July 21 and coincides with the observed low-energy ion event (middle panel in Fig. 2). Finally, event 5 occurred in a period with more stable plasma and IMF parameters, which may facilitate the scatter-free propagation of NR electrons toward SolO. 